\title{
Approaches to the Study of Drug-Induced Liver Injury
}

\author{
RJ Fontana ${ }^{1}$
}

Retrospective studies of administrative databases have led to highly variable estimates of the incidence and natural history of drug-induced liver injury (DILI) and the agents implicated. In contrast, prospective multicenter registry studies allow for more accurate phenotyping of individual patients with DILI, as well as for collection of biological samples for mechanistic studies. In addition to improved diagnostic and prognostic biomarkers, standardized causality assessment tools are needed, as well as population-based studies that represent the full spectrum of disease severity. This would facilitate further research into the pathogenesis of DILI, leading ultimately to the prevention of this condition.

The increased use of prescription medications has been associated with improvement in the general health, well being, and longevity of millions of people in the United States. However, a concomitant increase in the incidence of multiple serious adverse drug reactions (ADRs) has also been reported. Although drug-induced liver injury (DILI) is relatively uncommon (the estimated incidence related to the use of individual drugs ranges from 1 in 10,000 to 1 in 1,000,000 patient-years), DILI is the most common reason for drugs in development being denied regulatory approval and for others being withdrawn. ${ }^{1}$ Furthermore, the morbidity and mortality from DILI are substantial, with nearly $10 \%$ of the patients who develop jaundice from severe acute hepatocellular liver injury dying or requiring liver transplantation. ${ }^{2}$ Therefore, additional studies of the risk factors and mechanisms of DILI are urgently needed to allow otherwise effective drugs to be safely administered to the growing population of aging individuals.

\section{RECOGNITION AND DIAGNOSIS OF DILI}

The incidence of DILI is not well known, in part because of the lack of standardized and universally accepted diagnostic criteria. Given that DILI accounts for $<1 \%$ of American patients presenting with acute hepatitis and/or jaundice, clinicians must first exclude other more common etiologies of liver disease such as viral hepatitis (i.e., A, B, C), alcohol, pancreaticobiliary disease, hepatic ischemia, and autoimmune hepatitis. ${ }^{3,4}$ Also, DILI due to a particular drug can lead to differing phenotypes in individual patients and can mimic nearly all forms of acute and chronic liver disease. Finally, most cases of DILI are not related to the dose, route, or duration of treatment with the suspect medication and are not restricted to any readily identifiable group of high-risk patients. These challenges, coupled with the lack of a confirmatory laboratory biomarker, make DILI largely a diagnosis of exclusion based on "circumstantial evidence."

Several clinical features are commonly sought in attempting to evaluate the role of a medication in patients with otherwise unexplained acute and/or chronic liver disease (Table 1). ${ }^{5}$ Unfortunately, most DILI patients do not have readily recognizable hypersensitivity features such as rash or eosinophilia at presentation. In addition, diagnostic challenges arise if a patient is taking multiple medications, herbal or dietary supplements with multiple ingredients, or a newly approved drug without an established signature of hepatotoxicity. ${ }^{6}$ Patients with underlying liver disease who may be more susceptible to adverse outcomes can pose formidable diagnostic challenges. Finally, not all patients improve with dechallenge, and rechallenge is rarely, if ever, knowingly performed. ${ }^{7}$ Several causality assessment instruments have been used to help standardize the approach to DILI diagnosis. ${ }^{6}$ However, the currently available instruments, including expert opinion, have substantial inter- and intraindividual variability. ${ }^{8}$ Therefore, improved, evidence-based causality assessment methods and objective laboratory biomarkers of DILI are urgently needed.

\section{RETROSPECTIVE STUDIES OF DILI}

Several retrospective studies of DILI in the United States have described its incidence, the suspect medications, and patient outcomes. ${ }^{4,9}$ Many of these studies relied on a search of available medical records or administrative databases for either keywords or ICD-9 codes (from the International Classification of Diseases, 9th revision) associated with DILI. ${ }^{4,10}$ However, most of these ICD-9 codes have a low specificity $(1-5 \%)$, and the sensitivity of these approaches is 


\begin{tabular}{ll}
$\begin{array}{l}\text { Table } 1 \text { Diagnostic criteria for idiosyncratic drug-induced liver } \\
\text { injury }\end{array}$ & Comment \\
\hline Criterion & $\begin{array}{l}\text { Variable range with many drugs but } \\
\text { usually }<12 \text { months }\end{array}$ \\
\hline Time to onset & $\begin{array}{l}\text { Dechallenge is problematic in ALF } \\
\text { patients and in those who develop } \\
\text { chronic injury }\end{array}$ \\
\hline $\begin{array}{l}\text { Course of liver injury after drug } \\
\text { cessation }\end{array}$ & $\begin{array}{l}\text { Few bona fide clinical risk factors } \\
\text { have been established }\end{array}$ \\
\hline Risk factor(s) for hepatotoxicity & $\begin{array}{l}\text { Difficult to exclude when multiple } \\
\text { drugs or herbals are coadministered }\end{array}$ \\
\hline Concomitant hepatotoxic drugs & $\begin{array}{l}\text { Differ for hepatocellular and } \\
\text { cholestatic/mixed }\end{array}$ \\
\hline Exclusion of competing causes & $\begin{array}{l}\text { Problematic with newly approved } \\
\text { drugs and herbal products }\end{array}$ \\
\hline Prior reports of hepatotoxicity & $\begin{array}{l}\text { Rarely done and shows variable } \\
\text { results }\end{array}$ \\
\hline Rechallenge & $\begin{array}{l}\text { DILI can mimic nearly all known } \\
\text { forms of acute and chronic liver injury }\end{array}$ \\
\hline Liver histology & $\begin{array}{l}\text { Validated diagnostic biomarkers not } \\
\text { yet established }\end{array}$ \\
\hline Objective and confirmatory & laboratory test \\
\hline
\end{tabular}

ALF, acute liver failure; DILI, drug-induced liver injury.

limited because of the lack of a gold standard for diagnosis. The estimated incidence of DILI in these studies has been highly variable because of differences in case definition, coding accuracy, and the extent to which competing etiologies were excluded (i.e., $0.024 \%$ in a health maintenance organization population vs. $1 \%$ in hospitalized patients). ${ }^{11,12}$ Furthermore, the true incidence of DILI is probably underestimated, whereas its severity is probably overestimated from referral center studies given that the majority of DILI cases in the general population present as asymptomatic outpatients. ${ }^{13}$ Future studies of DILI epidemiology, merging laboratory, medication, and health-care claims databases and using the more robust and specific ICD-10 diagnostic coding, may prove informative for hypothesis generation regarding putative risk factors. However, pharmacoepidemiological inferences from retrospective studies of a rare and difficult-to-diagnose ADR like DILI should be interpreted with caution.

\section{POSTMARKETING SURVEILLANCE STRATEGIES}

The US Food and Drug Administration has tracked a multitude of ADRs for many years through the MedWatch voluntary reporting system. However, important diagnostic data are frequently missing or incomplete, and the ability to obtain follow-up information is limited. ${ }^{14}$ Furthermore, it is estimated that $<10 \%$ of all ADRs are reported to regulatory authorities, because of the reluctance of clinicians to report new ADRs. (In support of this, a recent prospective study demonstrated that the true incidence of DILI in the general population of France was nearly 16 -fold higher than that reported to regulatory authorities. ${ }^{13}$ ) Therefore, government registries have limited ability to accurately track the incidence, etiologies, and outcomes of DILI in the general population. Nonetheless, data mining of these databases can help to detect hepatotoxicity signals from existing products on the market and also generate hypotheses for risk factors. ${ }^{15}$

\section{PROSPECTIVE STUDIES \\ The DILI Network}

The DILI Network (DILIN) was established by the National Institutes of Health in 2003 to improve our understanding of the risk factors, natural history, and biological basis of DILI. Eight clinical sites along with a data-coordinating center provide the infrastructure to enroll pediatric and adult patients with liver injury that meets minimal laboratory criteria and is suspected to be due to any drug or herbal product taken within the 6 months before the onset of the injury. ${ }^{16}$ Patients with known fatty liver disease, chronic hepatitis B or C, or HIV infection are encouraged to participate.

At the baseline study visit, a detailed medical history is obtained, and a physical examination is carried out to exclude competing causes of liver injury (Table 2). In addition, blood, urine, peripheral lymphocytes, and DNA samples are collected for mechanistic studies. Available liver biopsy samples are reviewed by an expert hepatopathologist to create a library of diagnostic and prognostic histopathological features of wellcharacterized cases of DILI. All subjects with laboratory or radiological evidence of ongoing liver injury 6 months after the onset of DILI are followed so as to determine the outcome at 2 years. The DILIN prospective study also retains contact with patients for up to 20 years after enrollment, for participation in future mechanistic studies.

In order to promote awareness of the study and to facilitate recruitment, brochures and e-mails are sent to local physicians and related information is published in professional journals and posted at national meetings. A recent report of the first 300 patients enrolled in the DILIN prospective study showed that $60 \%$ were female and $70 \%$ were Caucasian; the mean age was 48 years (range: $2-81$ years). ${ }^{17}$ The mean serum alanine aminotransferase level was $788 \mathrm{IU} / \mathrm{l}$ at the onset of DILI, with a mean total bilirubin level of $11.4 \mathrm{mg} / \mathrm{dl}$. More than $50 \%$ of the subjects required hospitalization, and nearly $60 \%$ underwent liver biopsies.

Causality is determined by consensus expert opinion of three experienced hepatologists who independently review all the available clinical, laboratory, and diagnostic data as well as a clinical narrative. The majority of the first 300 DILIN patients had high causality scores, with $32 \%$ being definite, $41 \%$ very likely, and $14 \%$ probable. However, there were nine patients (3\%) with other more likely causes of liver injury, including unsuspected acute hepatitis $\mathrm{C}$ virus infection. The median duration of medication use was 42 days, with antibiotics (45\%) and central nervous system-acting drugs (15\%) being the most commonly implicated drugs. A single drug was implicated in DILI in $73 \%$ of the patients, whereas more than a single drug was suspected in $18 \%$ and a variety of herbal products in $9 \%$. The most commonly reported suspect drugs were amoxicillin/ clavulanate, followed by nitrofurantoin, isoniazid, and trimethoprim-sulfamethoxazole. 
Table 2 Visit schedule in the DILIN prospective study

\begin{tabular}{|c|c|c|c|}
\hline Item & Baseline visit & 6-Month study visit & 12-And 24-month study visits \\
\hline Eligible patients & All with suspected DILI & All with suspected DILI & Chronic DILI patients only ${ }^{a}$ \\
\hline Demographics & $X$ & & \\
\hline Pharmacy use & $x$ & & \\
\hline Medication compliance & $x$ & & \\
\hline Family history & $X$ & & \\
\hline Lifetime use of suspect medication & $X$ & & \\
\hline Diagnostic laboratory tests ${ }^{b}$ & $X$ & & \\
\hline HCV/HBV follow-up labs ${ }^{c}$ & $X$ & $x$ & \\
\hline $\begin{array}{l}\text { Concomitant medication and herbal } \\
\text { product use }\end{array}$ & $X$ & $x$ & $X$ \\
\hline Medical history & $X$ & $\mathrm{X}$ & $X$ \\
\hline Alcohol use and smoking & $X$ & $X$ & $X$ \\
\hline Physical examination & $X$ & $X$ & $X$ \\
\hline Symptom score & $X$ & $X$ & $x$ \\
\hline Quality-of-life survey & $X$ & $x$ & $X$ \\
\hline Standard laboratory tests & $x$ & $x$ & $X$ \\
\hline Research blood sample & $X$ & $x$ & $X$ \\
\hline Research urine sample & $x$ & $x$ & $x$ \\
\hline Liver imaging & $x$ & $x$ & $x$ \\
\hline
\end{tabular}

Anti-SmAb, anti-smooth muscle antibody; CMV, cytomegalovirus; DILI, drug-induced liver injury; DILIN, DILI Network; EBV, Epstein-Barr virus; HBV, hepatitis B virus; $\mathrm{HBsAg}$, hepatitis B surface antigen; HCV, hepatitis C virus; IgM, immunoglobulin M.

${ }^{a}$ Chronic DILI: evidence of persistent laboratory, radiologic, or pathologic abnormalities 6 months after onset of DILI. ${ }^{b} A l l$ subjects must have testing for anti-HAV (IgM), HBsAg, anti-HBC (IgM), anti-HCV, HCV RNA, ANA, anti-SmAb, and ceruloplasmin if age <50, AMA if mixed/cholestatic DILI, anti-CMV (IgM), or CMV-DNA by PCR, and EBV serologies or monospot test. ${ }^{c}$ Only in patients with known chronic HCV/HBV before onset of DILI.

Adapted from ref. 16.

\section{The Acute Liver Failure Study Group}

The Acute Liver Failure Study Group (ALFSG) is a consortium of academic centers funded by the National Institutes of Health to study the etiologies and outcomes of acute liver failure (ALF) in the United States. ALF is a dramatic and potentially lifethreatening clinical syndrome defined by the onset of coagulopathy (international normalized ratio $>1.5$ ) and changes in mental status within 8-26 weeks of the onset of the illness, with an estimated annual incidence of only 3,000 cases/year in the United States. ${ }^{18}$ All enrolled ALFSG patients have extensive clinical data and biological samples collected during their hospital stay. A review of the first 1,033 adult ALF patients enrolled between 1998 and 2007 demonstrated that acetaminophen overdose accounted for $45 \%$ of the cases and idiosyncratic DILI for $12 \% .{ }^{5}$ Three-week spontaneous survival was $64 \%$ in patients with acetaminophen overdose but only $26 \%$ in those with severe idiosyncratic DILI.

The mean age of the 133 ALF patients with idiosyncratic DILI was 44 years, and $71 \%$ of them were female. ${ }^{19}$ The median value of serum alanine aminotransferase at the time the patient presented with the condition was $574 \mathrm{IU} / \mathrm{l}$, and the median bilirubin value was $21 \mathrm{mg} / \mathrm{dl}$. The most frequently implicated agents were antituberculosis drugs (18\%), sulfa compounds (9\%), and phenytoin (7\%). Causality assessment by expert opinion classified $81 \%$ as highly likely and $15 \%$ as probable. ${ }^{19}$ Research challenges identified in this study include the inability to obtain a reliable medication history from the patient, the lack of improvement with dechallenge, and the absence of any unique diagnostic features in many of the DILI patients as compared with subjects with indeterminate ALF. However, all the enrolled patients have now been followed up for 2 years in order to determine long-term outcomes and for enrollment in additional mechanistic studies.

\section{United Network of Organ Sharing database}

The indications and outcomes of all patients in the United States undergoing solid organ transplantation are tracked by the United Network of Organ Sharing (UNOS). A recent review of 141 adults with ALF due to DILI who underwent liver transplantation between 1992 and 2001 showed a preponderance of women $(76 \%){ }^{20}$ The most commonly implicated drugs were isoniazid (17\%), propylthiouracil (9\%), phenytoin (7\%), and valproate (7\%). These findings, and those of the ALFSG, suggest that several classes of commonly used drugs (such as antibiotics and anticonvulsants) are the most frequent causes of severe DILI in the United States. However, the limitations of the UNOS database include a lack of information regarding the indication for which the suspect medication was prescribed, how a diagnosis was established, and the duration of use of the medication.

\section{FUTURE STUDIES}

Continued enrollment of patients into prospective registry studies such as the DILIN initiative and others should help to improve our understanding of the incidence and etiologies of idiosyncratic 
DILI. However, additional population-based studies are also needed to define the full spectrum of severity of DILI in the general population. True prospective studies that track a large group of patients receiving a drug such as isoniazid, from the time of initial exposure until the onset of liver injury, would provide important information regarding clinical, environmental, and dietary risk factors. However, prospective monitoring of a cohort of several thousand treated patients for an ADR that occurs in $<1 \%$ of exposed individuals requires tremendous resources.

The value of collecting biological samples for mechanistic studies from individual patients with bona fide DILI was recently demonstrated in a case-control study of patients with flucloxacillin-related cholestasis. ${ }^{21}$ In that study, an 80 -fold increased risk of DILI was attributable to a single-nucleotide polymorphism in the HLA-B ${ }^{\star} 5701$ locus, which was confirmed in a validation cohort. This striking proof-of-concept study demonstrates the potential value of modern genomics, transcriptomics, and proteomics in unraveling the mechanism of rare ADRs such as DILI. The development of prospective DILI registries in countries that utilize similar recruitment, eligibility, and causality assessment methods (e.g., Spain, Japan, and Korea) will prove invaluable for validating future diagnostic and prognostic biomarkers of DILI. ${ }^{22}$ It is hoped that these efforts will ultimately provide clinicians with the tools and knowledge to better follow the Hippocratic oath of "Do No Harm" by maximizing efficacy and minimizing the incidence of unintended ADRs such as DILI.

\section{ACKNOWLEDGMENTS}

The author was supported by National Institutes of Health grants R01-DK-58369-01 (ALFSG) and U01 DK065184-01 (DILIN). The author would like to acknowledge Portia Bonner for help with manuscript editing and submission.

\section{CONFLICT OF INTEREST}

The author has consulted for or received payments from Abbott Labs, GlaxoSmithKline, Vertex Consulting, Roche Labs, Gilead, and the BristolMyers Squibb Speakers'Bureau.

○ 2010 American Society for Clinical Pharmacology and Therapeutics

1. Temple, R.J. \& Himmel, M.H. Safety of newly approved drugs: implications for prescribing. JAMA 287, 2273-2275 (2002).

2. Andrade, R.J. et al. Drug-induced liver injury: an analysis of 461 incidences submitted to the Spanish registry over a 10-year period. Gastroenterology 129, 512-521 (2005).
3. Galan, M.V., Potts, J.A., Silverman, A.L. \& Gordon, S.C. The burden of acute nonfulminant drug-induced hepatitis in a United States tertiary referral center [corrected]. J. Clin. Gastroenterol. 39, 64-67 (2005).

4. Vuppalanchi, R., Liangpunsakul, S. \& Chalasani, N. Etiology of new-onset jaundice: how often is it caused by idiosyncratic jaundice in the United States? Am. J. Gastroenterol. 101, 1-5 (2006).

5. Fontana, R.J. Acute liver failure due to drugs. Semin. Liver Dis. 28, 175-187 (2008).

6. Hayashi, P.H. Causality assessment in drug-induced liver injury. Semin. Liver Dis. 29, 348-356 (2009).

7. Papay, J.I. et al. Drug-induced liver injury following positive drug rechallenge. Regul. Toxicol. Pharmacol. 54, 84-90 (2009).

8. Rochon, J. et al. Reliability of the Roussel Uclaf Causality Assessment Method for assessing causality in drug-induced liver injury. Hepatology $\mathbf{4 8 ,}$ 1175-1183 (2008).

9. Bell, L.N. \& Chalasani, N. Epidemiology of idiosyncratic drug-induced liver injury. Semin. Liver Dis. 29, 337-347 (2009).

10. Jinjuvadia, K., Kwan, W. \& Fontana, R.J. Searching for a needle in a haystack: use of ICD-9-CM codes in drug-induced liver injury. Am. J. Gastroenterol. 102, 2437-2443 (2007).

11. Meier, Y. et al. Incidence of drug-induced liver injury in medical inpatients. Eur. J. Clin. Pharmacol. 61, 135-143 (2005).

12. Duh, M.S., Walker, A.M. \& Kronlund, K.H. Jr. Descriptive epidemiology of acute liver enzyme abnormalities in the general population of central Massachusetts. Pharmacoepidemiol. Drug Saf. 8, 275-283 (1999).

13. Sgro, C. et al. Incidence of drug-induced hepatic injuries: a French populationbased study. Hepatology 36, 451-455 (2002).

14. Moore, T.J., Cohen, M.R. \& Furberg, C.D. Serious adverse drug events reported to the Food and Drug Administration, 1998-2005. Arch. Intern. Med. 167, 1752-1759 (2007).

15. US Food and Drug Administration. Center for Drug Evaluation and Research. Guidance for Industry. Drug Induced Liver Injury: Premarketing Clinical Evaluation <http://www.fda.gov/downloads/Drugs/ GuidanceComplianceRegulatorylnformation/Guidances/UCM174090.pdf> (2009).

16. Fontana, R.J. et al.; DILIN Study Group. Drug-Induced Liver Injury Network (DILIN) prospective study: rationale, design and conduct. Drug Saf. 32, 55-68 (2009).

17. Chalasani, N. et al. Causes, clinical features, and outcomes from a prospective study of drug-induced liver injury in the United States. Gastroenterology 135 , 1924-1934, 1934.e1 (2008).

18. Ostapowicz, G. et al. Results of a prospective study of acute liver failure at 17 tertiary care centers in the United States. Ann. Intern. Med. 137, 947-954 (2002).

19. Reuben, A., Koch, D.G. \& Lee, W.M. Acute liver failure secondary to drug induced liver injury: causes and consequences. (Abstract). Hepatology 54, A\#92 (2009).

20. Russo, M.W., Galanko, J.A., Shrestha, R., Fried, M.W. \& Watkins, P. Liver transplantation for acute liver failure from drug induced liver injury in the United States. Liver Transpl. 10, 1018-1023 (2004).

21. Daly, A.K. et al. HLA-B*5701 genotype is a major determinant of drug-induced liver injury due to flucloxacillin. Nat. Genet. 41, 816-819 (2009).

22. Fontana, R.J. et al. Standardization of nomenclature and causality assessment in drug-induced liver injury: summary of a clinical research workshop. Hepatology (in press). 